\title{
Engagement and communication among participants in the ClinSeq Genomic Sequencing Study
}

\author{
Gillian W. Hooker, PhD, ScM ${ }^{1,4}$, Kendall L. Umstead, MS', Katie L. Lewis, MS², Laura K. Koehly, PhD³, \\ Leslie G. Biesecker, MD² and Barbara B Biesecker, PhD, MS ${ }^{1}$
}

Purpose: As clinical genome sequencing expand its reach, understanding how individuals engage with this process are of critical importance. In this study, we aimed to describe internal engagement and its correlates among a ClinSeq cohort of adults consented to genome sequencing and receipt of results.

Methods: This study was framed using the precaution adoption process model (PAPM), in which knowledge predicts engagement and engagement predicts subsequent behaviors. Prior to receipt of sequencing results, 630 participants in the study completed a baseline survey. Engagement was assessed as the frequency with which participants thought about their participation in ClinSeq since enrollment.
Results: Results were consistent with the PAPM: those with higher genomics knowledge reported higher engagement $(r=0.13$, $P=0.001)$ and those who were more engaged reported more frequent communication with their physicians $(r=0.28, P<0.001)$ and family members $(r=0.35, P<0.001)$ about ClinSeq. Characteristics of those with higher engagement included poorer overall health $(r=-0.13$, $P=0.002)$, greater seeking of health information $(r=0.16, P<0.001)$, and more recent study enrollment $(r=-0.21, P<0.001)$.

Conclusion: These data support the importance of internal engagement in communication related to genomic sequencing.

Genet Med advance online publication 20 October 2016

\section{INTRODUCTION}

Large-scale clinical sequencing is changing, and in some cases up-ending, medical paradigms. ${ }^{1,2}$ The traditional pathway to diagnosis of genetic disease may be reversed, with mutations being identified prior to canonical symptoms or family history. As methods for delivery of genome-based information are developed, it is important to understand how individuals engage cognitively and emotionally with this information and how an individual's degree of internal engagement drives decisions about how to apply genetic information.

Genetic tests can, but do not necessarily, predict behavior. For example, women found to have mutations in the BRCA1 and $B R C A 2$ genes are significantly more likely to undergo prophylactic oophorectomy and mastectomy than women with a family or personal history of breast or ovarian cancer in whom mutations are not identified. ${ }^{3}$ Learning one has an APOE $\varepsilon 4$ allele and is at elevated risk for Alzheimer disease is associated with the decision to purchase long-term-care insurance. ${ }^{4}$ Moreover, individuals who are found to carry mutations in CFTR subsequently communicate these results to family members. ${ }^{5}$ However, in each of these examples, behaviors are not consistently adopted. Furthermore, receiving genetic risk information does not seem to promote sustained changes in lifestyle-related health behaviors such as quitting smoking, adopting a healthy diet, or exercising regularly. ${ }^{6-8}$ It seems likely that contextual factors and individual differences may be important in motivating change if it is to occur. Reported predictors of behavior following genetic testing include worry about the disease, perceived efficacy of the behavior in affecting a desired outcome, and family experience with illness and disease. ${ }^{4,5,9}$ It remains to be seen how these effects may or may not be different when genetic information is provided outside of a specific disease or family history context or in the context of information about multiple different genes related to various phenotypes.

In the literature, when theory is applied to understanding motivations and perceptions of genetic tests, genetic testing is often framed as a stressor, thus casting testing in an inherently negative light. ${ }^{10}$ However, several meta-analyses focusing primarily on cancer susceptibility testing have found that genetic testing does not significantly impact psychological well-being, as measured with traditional measures of patient states like depressive symptoms and anxiety. ${ }^{11,12}$ In recent years, measures have been developed to specifically assess outcomes of genetic testing. ${ }^{10,13}$ The Multidimensional Impact of Cancer Risk Assessment (MICRA) is one such scale. This scale has excellent discriminative validity, with patients who have BRCA1/2 mutations having much higher scores than those who do not have BRCA1/2 mutations. One study included the MICRA in an assessment of the effectiveness of a decision aid for helping 
patients with a $B R C A 1 / 2$ mutation reach a decision regarding management of their risk. In this study, the decision aid was found to be effective; it helped undecided women reach a decision, thus reducing their decisional conflict and increasing their decisional satisfaction..$^{14}$ In ancillary analyses, it was noted that use of the decision aid was also associated with higher MICRA scores. ${ }^{15}$ This led to the hypothesis that the MICRA may be capturing internal engagement, which is potentially necessary for systematic processing of one's genetic risk and useful for effective medical decision making.

The idea that internal engagement predicts effective decision making is consistent with the precaution adoption process model (PAPM) ${ }^{16}$ As framed in this model, internal engagement is the amount of time an individual devotes to thoughts about a particular message, event, or stimulus. ${ }^{17}$ Internal engagement encompasses both positive and negative thoughts, similar to the positive and negative responses to genetic testing conceptualized in the MICRA. ${ }^{10,18}$ It also covers both cognitive and emotional processes and deliberate and "intrusive" thinking. ${ }^{10}$ Within the PAPM, engagement is a stage required for active decision making. ${ }^{16}$

The PAPM is a stage-based model that defines decision states with regard to a particular behavior and allows for discrimination between individuals who are actively making decisions about health information and health behaviors and those who are either unaware that decisions need to be made or unengaged with the process of making decisions. ${ }^{17}$ This model is particularly useful in guiding intervention development targeted to stages of awareness (e.g., unaware, unengaged, undecided, decided for, or decided against). Specifically, stage-matched interventions have been proposed for guiding decision making about breast and colon cancer screening, ${ }^{19,20}$ prenatal testing, ${ }^{18}$ calcium supplementation, ${ }^{21}$ and parent-child safety behaviors. ${ }^{22}$ An additional strength of this model is the ease with which it can be combined with other models of health decision making, ${ }^{16,20}$ thereby framing more complex models of decision making that may operate on assumptions of knowledge and internal engagement.

The PAPM provides a structure in which to understand the relationships between knowledge, internal engagement, decision making regarding behavior, and ultimate behavior (see Figure 1). This combined model is well suited to studying the decision-making processes regarding new technologies, such as genome sequencing, about which knowledge and engagement may be variable. In many clinical genomic sequencing environments, motivators related to the primary reason for seeking

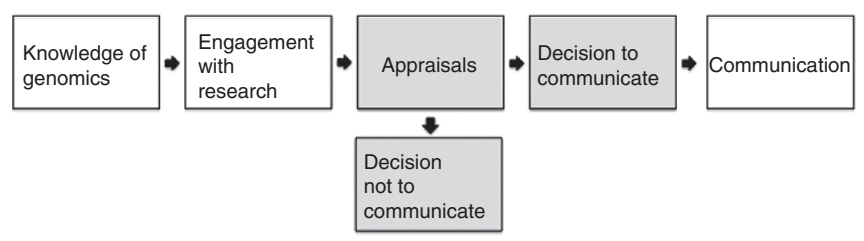

Figure 1 Application of the precaution adoption model to participation in ClinSeq. Constructs presented in the white boxes were measured for the analyses presented in this study. sequencing may lead to variable levels of engagement with any specific decision. Patients and research participants entering sequencing with highly salient health concerns or poorer health may be more internally engaged..$^{23}$ Similarly, patients who have a tendency to seek health information in general are likely to be more engaged in the clinical sequencing process. ${ }^{24} \mathrm{~A}$ potential strength of this model is that it can be used to inform stage-tailored interventions to deliver sequencing information in a way that is most useful to patients undergoing sequencing. To date, although patient engagement is frequently discussed as a critical component of patient-centered research and medical practice, ${ }^{25,26}$ internal engagement as a cognitive and affective patient state has not been well explored.

This paper aims to describe internal engagement in the context of a genome sequencing research study. Guided by the PAPM, we demonstrate the predicted relationships of knowledge and communication with engagement. Further, we provide evidence for validity of engagement as a construct relevant to genetics, showing that engagement behaves as would be predicted by our definition. Finally, we elaborate on communication patterns observed in the cohort about participation in the ClinSeq study.

\section{MATERIALS AND METHODS}

Participants were recruited from the larger NIH ClinSeq study and asked to complete a baseline survey on health cognitions, affect, personality traits, and health-related behaviors prior to receipt of any genome sequencing results. Results from this survey were collected from August 2012 to April 2015 from participants recruited between 2007 and 2015. The details of participant recruitment, enrollment, and survey delivery are described elsewhere. ${ }^{27}$

\section{Measures}

Knowledge was measured using a 16-item knowledge scale assessing participant knowledge about the benefits, limitations, and applications of genome sequencing. ${ }^{28}$

Internal engagement was measured as the following singleitem question: "How often do you think about your participation in the ClinSeq Study?" Participants were asked to respond on a scale of $1-5$, with 1 indicating never; 2 , yearly; 3 , monthly; 4, weekly; and 5, daily.

Communication was assessed in two ways. First, we asked participants to rate on a scale of 1-5 (with 1 representing not at all and 5 representing very often) how often they talk with their doctor, family, or friends about their participation in ClinSeq. Each of these questions was analyzed individually; all were normally distributed across the sample. Second, we asked participants to report whom they have told about their participation in ClinSeq; we asked about specific family members individually (spouse, biological mother, biological father, biological daughter, biological son, sister, or brother) and controlled for whether the participants have these relatives and whether they are living. We also allowed participants to add additional relatives with whom they communicated. For spouses and parents, 


\section{ORIGINAL RESEARCH ARTICLE}

we report the percent of participants who told that relative from the total number of participants who reported having that family member in their lives currently. For offspring and siblings, we asked participants to tell us how many relatives they have and, of those, how many they told, and then calculated "percent communicated to" and report the mean percent communicated to for the sample.

Several constructs hypothesized to correlate with internal engagement were also assessed, including global health, healthcare utilization, health-information seeking, education, and length of time since initial enrollment in ClinSeq. We assessed participant global health using the 10- item PROMIS global health scale (http://www.nihpromis.org). The Cronbach's alpha for this scale was 0.87 . Our measure of frequency of doctor's visits was derived from the question "How many times have you seen your primary-care doctor in the last year for any reason?" The response scale was from 1 to $4: 1$, not at all; 2 , once; 3 , twice; and 4, more than twice. To assess health-information seeking, we asked, "In the past 30 days, how often would you say you have actively looked for information about ways to stay healthy and feel better?" Highest degree earned was collected as a part of the demographic information. Time since enrollment was calculated as the difference between enrollment and survey completion.

\section{Statistical analyses}

All analyses were completed using IBM SPSS Statistics version 22 (Armonk, NY). For univariate variables, means, standard deviations, medians, and frequency distributions were calculated. Bivariate correlations are described using Pearson's $r$, with a Holm correction to control for the family-wise error rate. ${ }^{29}$ To compare means, $t$-tests were used.

\section{Ethics statement}

This research was reviewed and approved by the National Human Genome Research Institute Institutional Review Board at the National Institutes of Health. Participants signed written informed consent for the overall protocol at the time of enrollment. The survey stated that participant consent to participate was implicit in their completion of the survey, which

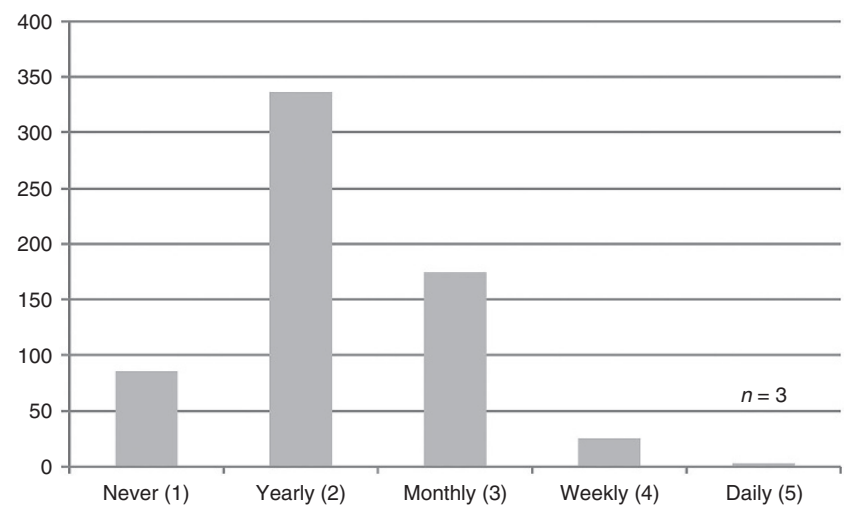

Figure 2 Engagement in the ClinSeq sample: frequency of thoughts about ClinSeq $(\boldsymbol{n}=\mathbf{6 2 4})$. Numeric mean: $2.2 ;$ SD: $0.7 ; n=3$. was approved by the National Human Genome Institute Institutional Review Board. Survey data were de-identified before analysis.

\section{Demographics}

\section{RESULTS}

The majority of participants were college-educated $(89.2 \%)$ and white (92.5\%), and approximately half had been enrolled in the study for more than 4 years $(54.0 \%)$, with a mean time since enrollment of 4 years. The characteristics of the ClinSeq sample and subset of participants $(n=630)$ who completed the ClinSeq baseline survey have been described in detail. ${ }^{27}$

\section{Engagement}

The frequency with which ClinSeq participants thought about their participation was normally distributed (see Figure 2), with most participants (336) thinking about the study on a yearly basis. Eighty-six participants reported never thinking about ClinSeq and three participants reported thinking about ClinSeq on a daily basis.

Engagement, knowledge, and communication. Knowledge and engagement were significantly correlated $(r=0.128$, $P=0.005$ ) (see Table 1). Additionally, engagement was significantly correlated with frequency of communication about ClinSeq with doctors $(r=0.281, P=0.006)$, family members $(r=0.353, P=0.006)$, and friends $(r=0.353, P=0.007)$.

Engagement, health, health-related behaviors, and background. Individuals who reported better overall health were less likely to have high internal engagement $(r=-0.126, P=0.017)$ (see Table 1). More frequent seeking of health information $(r=0.158, P=0.008)$ and having enrolled more recently in the ClinSeq study (time since enrollment: $r=-0.205, P=0.01$ ) were similarly significantly associated with engagement. Seeing doctors more frequently $(r=0.093, P=0.025)$ and having

\section{Table 1 Correlates of engagement with ClinSeq}

\begin{tabular}{lrr} 
& Pearson's $\boldsymbol{r}$ & $\boldsymbol{P}^{\mathrm{a}}$ \\
\hline $\begin{array}{l}\text { Precaution adoption constructs } \\
\text { Knowledge }\end{array}$ & 0.128 & 0.001 \\
Communication with doctors & 0.281 & $<0.001$ \\
Communication with family & 0.353 & $<0.001$ \\
Communication with friends & 0.353 & $<0.001$ \\
Number of relatives told & 0.193 & $<0.001$ \\
Face validity constructs & & \\
Better overall health & -0.126 & 0.002 \\
More frequent seeking of health information & 0.158 & $<0.001$ \\
Time since enrollment & -0.205 & $<0.001$ \\
More frequent doctor visits & 0.093 & 0.021 \\
Have undergone genetic testing & 0.093 & 0.021 \\
\hline a Holm correction was applied to control for the family-wise error rate, which \\
yields a spectrum of significance thresholds ranging from an uncorrected value to \\
the conservative Bonferroni. Because all values remained significant, this resulted \\
in no difference in the manner in which the results were interpreted.
\end{tabular}

Volume 19 | Number 1 | January 2017 | GENETICS in MEDICINE 
undergone genetic testing in the past $(r=0.093, P=0.05)$ were also significantly associated with higher engagement.

Engagement and communication to specific relatives. We not only detected a significant relationship between the number of relatives told about ClinSeq and engagement $(r=0.193$, $P<0.001)$ but also observed differences in communication about ClinSeq by relationship (see Table 2). Most married participants $(85.7 \%, n=540)$ told their spouses or partners about their participation in ClinSeq $(87.2 \%, n=471)$. Fifty-five percent of participants $(n=506)$ communicated their participation in ClinSeq to at least one daughter; $51 \%(n=523)$ told at least one son; $53 \%(n=531)$ told at least one sister; and $47 \%(n=542)$ told at least one brother. Of participants with relatives available to tell, participants were significantly more likely to tell their children than their siblings $(t=6.835, P<0.001)$ and more likely to tell their sisters than brothers $(t=5.804, P<0.001)$.

\section{Discussion}

\section{DISCUSSION AND CONCLUSIONS}

Although the concept of engagement arises frequently in patientcentered outcomes research, little consensus exists regarding how it is defined or measured. In this study, we assessed the internal engagement process experienced by individuals participating in a genome sequencing study. Using the PAPM as a guide, we demonstrated theoretically predicted relationships between knowledge about genome sequencing, engagement with genome sequencing, and communication about genome sequencing. Internal engagement among ClinSeq participants was associated with poorer overall health, information seeking, and more recent enrollment in the study (Table 1 ).

Engagement of research participants and patients is a critical element for realizing the full potential of genomic medicine. The premise that "actionable" genomic variants will provide health benefits to those who learn about them rests on the assumption that they will act on that information. The PAPM provides a useful framework for understanding why research participants and patients might not act on such information and guides

Table 2 Communication with family members about participation

\begin{tabular}{lcc} 
Relationship & $\begin{array}{c}\text { Percent having at } \\
\text { least one living } \\
\text { relation }\end{array}$ & $\begin{array}{c}\text { Percent of } \\
\text { available relatives } \\
\text { communicated to }\end{array}$ \\
\hline $\begin{array}{l}\text { Spouse } \\
\text { Children } \\
\quad \text { Biological son(s) }\end{array}$ & $86 \%$ & $87 \%$ \\
$\quad$ Biological daughter(s) & $60 \%$ & $51 \%$ \\
$\begin{array}{l}\text { Parents } \\
\quad \text { Mother }\end{array}$ & $61 \%$ & $55 \%$ \\
$\quad$ Father & $43 \%$ & $60 \%$ \\
Siblings & $25 \%$ & $55 \%$ \\
$\quad$ Biological sister(s) & $68 \%$ & \\
$\quad$ Biological brother(s) & $72 \%$ & $53 \%$ \\
Physicians & $\mathrm{N} / \mathrm{A}$ & $47 \%$ \\
\hline
\end{tabular}

development of interventions in the event that they do not. ${ }^{16}$ Specifically, some test results may be highly salient and significant enough to an individual to produce sustained internal engagement and hence a greater likelihood of behavior change. Alternatively, other types of results, those holding less salience, those associated with mild or moderate risk increases, those in the absence of family history, and/or those delivered in a onetime clinical communication event may not produce sufficient internal engagement to promote behavior change. Low internal engagement might be one explanation for the consistent finding that genetic test results do not promote sustained changes in diet, exercise, or smoking behavior. ${ }^{6}$ Clinical sequence data are anticipated to become a reference, rather than a single test, that can be accessed over years and inform health care longitudinally across life stages. ${ }^{30}$ Patient engagement is a critical component of interaction with providers about their sequence as they age.

The PAPM also holds several advantages over other models used previously to study the genetic testing process. Stress and coping models have frequently been applied in the study of individuals coping with genetic risk, although their utility in a genomic testing setting is unclear. ${ }^{31-33}$ These models provide a useful framework to describe the varying ways that tests can be appraised (e.g., risky, having negative consequences, or controllable) and the ways that appraisals can predict coping responses and subsequent outcomes. Yet they tend to cast testing in an inherently negative light, de-emphasizing positive or neutral aspects of the experience. ${ }^{10}$ The PAPM provides a more neutral framework to study individual decisions following genetic testing. Further, the PAPM, with the central concept of engagement, accounts for the magnitude of impact that a result may have on an individual. Finally, the PAPM can be easily integrated with other decision-making models when framing future studies. ${ }^{20}$

Recognizing that the individuals in this baseline study have not yet received information about their sequence variants, we studied communication about enrollment to capture actions potentially driven by knowledge and subsequent engagement. Individuals with less knowledge about genome sequencing were less likely to be engaged with the study and less likely to have communicated with others about the study. If an important goal of ClinSeq includes increased communication by participants, then it will be important to determine whether noncommunication is due to a lack of knowledge or lack of engagement. Education might be beneficial for those who lack knowledge about sequencing, but it may be ineffective for those who are not engaged with ClinSeq or for whom other issues (e.g., major health problems, financial problems, job stress) outcompete the idea of talking to others about ClinSeq. ${ }^{17}$ The fact that most participants thought about ClinSeq on a yearly basis is not surprising because in the time leading up to this survey participants received, at a minimum, biannual contact by the study team to update them on the course of the study.

Although the decision to communicate about enrollment in a sequencing study is probably a value-based decision and is itself unlikely to lead to any particular positive (or negative) 


\section{ORIGINAL RESEARCH ARTICLE}

health outcome for the participant or their family members, it is interesting to note that communication is relatively high and most participants have communicated at least once with most of their immediate family members about their enrollment. Our findings are consistent with the communication behaviors of ClinSeq participants interviewed after receiving results. ${ }^{34}$ On some level, this is not surprising given the enthusiasm with which participants entered the study ${ }^{35}$ and the optimism they have expressed regarding the downstream benefits of genomics and genomics research to future generations ${ }^{36}$ It is also encouraging because of the likelihood that they will acquire information in the future that may have more direct implications for their family members. ${ }^{1}$ It is notable that participants were more likely to talk to sisters about enrollment than brothers, as has been reported in other studies, ${ }^{37,38}$ and it will be of interest to determine if this remains the case when actionable variants are returned.

\section{Conclusion}

It will be important to test the relationships described in this study in longitudinal studies with more normative decisions. For example, we hypothesize that engaged patients who learn of actionable risk variants for cardiac disease or cancer are more likely to act quickly on the recommendations accompanying those variants (e.g., will be quicker in scheduling consults and screening appointments) than patients who are not engaged. Further, exploration of internal engagement in populations less likely to be engaged is warranted. As an example, a parent's active internal engagement in caregiving of a child with a rare disease may outcompete engagement about their own risks if they learn of them via a secondary finding in genome sequencing. Finally, recognizing our conceptualization of engagement as potentially including negative or intrusive thoughts, it is possible that very high levels of engagement may be associated with normatively negative outcomes, including overutilization of health care and negative psychological states. It is also important to note that the measure of internal engagement used in this study is a single-item measure drawn directly from the study definition of engagement (the amount of time dedicated to thoughts about a particular subject). A multi-item measure could further differentiate the internal engagement levels of the sample and enable better characterization of those on the very high and low ends of the spectrum.

Inappropriate assumptions on the part of the provider about why a patient or research participant chooses not to follow up on recommended activities are barriers to good health care. By exploring their processes in decision making and delivering tailored interventions, we are more likely to achieve positive outcomes.

\section{Practice implications}

The findings of this study indicate that the more knowledgeable an individual is about a subject, the more likely they are to be internally engaged with it, and that internally engaged individuals are more likely to communicate with their family members. In clinical situations in which family communication is a desired outcome, individuals who lack knowledge or internal engagement may be at risk for poor communication with family members. Interventions designed to improve communication should assess these factors in order to maximize opportunities to promote effective use of genomic information.

\section{ACKNOWLEDGMENTS}

The authors thank the dedicated ClinSeq participants who completed the baseline survey. This research was funded by the Intramural Research Program of the National Human Genome Research Institute, National Institutes of Health. An earlier version of this analysis was presented by $\mathrm{Dr}$ Hooker at the National Society of Genetic Counselors in Anaheim, California, October 2013.

\section{DISCLOSURE}

K.L.U., L.K.K., K.L.L., and B.B.B. have no conflicts of interest to declare. L.G.B. is an uncompensated consultant to the Illumina Corporation and receives royalties from the Genentech and Amgen Corporations. G.W.H. is a full-time employee of NextGxDx.

\section{REFERENCES}

1. Johnston JJ, Rubinstein WS, Facio FM, et al. Secondary variants in individuals undergoing exome sequencing: screening of 572 individuals identifies highpenetrance mutations in cancer-susceptibility genes. Am J Hum Genet 2012;91:97-108.

2. Sloan JL, Johnston JJ, Manoli I, et al.; NIH Intramural Sequencing Center Group. Exome sequencing identifies ACSF3 as a cause of combined malonic and methylmalonic aciduria. Nat Genet 2011;43:883-886.

3. Schwartz MD, Isaacs C, Graves KD, et al. Long-term outcomes of BRCA1/ BRCA2 testing: risk reduction and surveillance. Cancer 2012;118: 510-517.

4. Zick CD, Mathews CJ, Roberts JS, Cook-Deegan R, Pokorski RJ, Green RC Genetic testing for Alzheimer's disease and its impact on insurance purchasing behavior. Health Aff (Millwood) 2005;24:483-490.

5. Ormond KE, Mills PL, Lester LA, Ross LF. Effect of family history on disclosure patterns of cystic fibrosis carrier status. Am J Med Genet C Semin Med Genet 2003;119C:70-77.

6. Hollands GJ, French DP, Griffin SJ, et al. The impact of communicating genetic risks of disease on risk-reducing health behaviour: systematic review with metaanalysis. BMJ 2016;352:i1102.

7. Marteau TM, French DP, Griffin SJ, Prevost AT, Sutton S, Watkinson C, et al. Effects of communicating DNA-based disease risk estimates on riskreducing behaviours. Cochrane Database Syst Rev 2010:CD007275. doi:10.1002/14651858.CD007275.pub2.

8. McBride CM, Koehly LM, Sanderson SC, Kaphingst KA. The behavioral response to personalized genetic information: will genetic risk profiles motivate individuals and families to choose more healthful behaviors? Annu Rev Public Health 2010;31:89-103.

9. Graves KD, Peshkin BN, Halbert CH, DeMarco TA, Isaacs C, Schwartz MD. Predictors and outcomes of contralateral prophylactic mastectomy among breast cancer survivors. Breast Cancer Res Treat 2007;104:321-329.

10. Cella D, Hughes $C$, Peterman A, et al. A brief assessment of concerns associated with genetic testing for cancer: the Multidimensional Impact of Cancer Risk Assessment (MICRA) questionnaire. Health Psychol 2002;21:564-572.

11. Braithwaite D, Emery J, Walter F, Prevost AT, Sutton S. Psychological impact of genetic counseling for familial cancer: a systematic review and meta-analysis. Fam Cancer 2006;5:61-75.

12. Hamilton JG, Lobel M, Moyer A. Emotional distress following genetic testing for hereditary breast and ovarian cancer: a meta-analytic review. Health Psychol 2009;28:510-518.

13. Esplen MJ, Stuckless N, Hunter J, et al. The BRCA Self-Concept Scale: a new instrument to measure self-concept in BRCA1/2 mutation carriers. Psychooncology 2009;18:1216-1229. 
14. Schwartz MD, Valdimarsdottir HB, DeMarco TA, et al. Randomized trial of a decision aid for BRCA1/BRCA2 mutation carriers: impact on measures of decision making and satisfaction. Health Psychol 2009;28:11-19.

15. Hooker GW, Leventhal KG, DeMarco T, et al. Longitudinal changes in patient distress following interactive decision aid use among BRCA1/2 carriers: a randomized trial. Med Decis Making 2011;31:412-421.

16. Weinstein ND. The precaution adoption process. Health Psychol 1988;7: 355-386.

17. Weinstein ND, Sandman PM. A model of the precaution adoption process: evidence from home radon testing. Health Psychol 1992;11:170-180.

18. Fransen MP, Essink-Bot ML, Oenema A, Mackenbach JP, Steegers EA, Wildschut HI. Ethnic differences in determinants of participation and non-participation in prenatal screening for Down syndrome: a theoretical framework. Prenat Diagn 2007;27:938-950.

19. LaPelle N, Costanza ME, Luckmann R, Rosal MC, White MJ, Stark JR. Staging mammography nonadherent women: a qualitative study. J Cancer Educ 2008;23:114-121.

20. Glanz K, Steffen AD, Taglialatela LA. Effects of colon cancer risk counseling for first-degree relatives. Cancer Epidemiol Biomarkers Prev 2007;16:1485-1491.

21. Blalock SJ, DeVellis RF, Giorgino KB, et al. Osteoporosis prevention in premenopausal women: using a stage model approach to examine the predictors of behavior. Health Psychol 1996;15:84-93.

22. Gielen AC, McKenzie LB, McDonald EM, et al. Using a computer kiosk to promote child safety: results of a randomized, controlled trial in an urban pediatric emergency department. Pediatrics 2007;120:330-339.

23. Nakash RA, Hutton JL, Lamb SE, Gates S, Fisher J. Response and non-response to postal questionnaire follow-up in a clinical trial-a qualitative study of the patient's perspective. J Eval Clin Pract 2008;14:226-235.

24. Iverson SA, Howard KB, Penney BK. Impact of internet use on health-related behaviors and the patient-physician relationship: a survey-based study and review. J Am Osteopath Assoc 2008;108:699-711.

25. Williams JK, Daack-Hirsch S, Driessnack M, Downing NR, Simon C. The importance of patient engagement. Genet Test Mol Biomarkers 2013;17:649.

26. Gruman J, Rovner MH, French ME, et al. From patient education to patient engagement: implications for the field of patient education. Patient Educ Couns 2010;78:350-356.
27. Lewis KL, Han PK, Hooker GW, Klein WM, Biesecker LG, Biesecker BB Characterizing participants in the ClinSeq genome sequencing cohort as early adopters of a new health technology. PLoS One 2015;10:e0132690.

28. Kaphingst KA, Facio FM, Cheng MR, et al. Effects of informed consent for individual genome sequencing on relevant knowledge. Clin Genet 2012:82:408-415

29. Olejnik S, Li J, Supattathum S, Huberty CJ. Multiple testing and statistical power with modified Bonferroni procedures. J Educ Behav Stat 1997;22: 389-406.

30. Biesecker LG. Opportunities and challenges for the integration of massively parallel genomic sequencing into clinical practice: lessons from the ClinSeq project. Genet Med 2012;14:393-398.

31. Gooding HC, Linnenbringer EL, Burack J, Roberts JS, Green RC, Biesecker BB. Genetic susceptibility testing for Alzheimer disease: motivation to obtain information and control as precursors to coping with increased risk. Patient Educ Couns 2006:64:259-267.

32. Shiloh S. Illness representations, self-regulation, and genetic counseling: a theoretical review. J Genet Couns 2006;15:325-337.

33. van Oostrom I, Meijers-Heijboer H, Duivenvoorden HJ, et al. The common sense model of self-regulation and psychological adjustment to predictive genetic testing: a prospective study. Psychooncology 2007;16:1121-1129.

34. Lewis KL, Hooker GW, Connors PD, Hyams TC, Wright MF, Caldwell S, et al. Participant use and communication of findings from exome sequencing: a mixed-methods study. Genet Med 2016;18:577-583.

35. Facio FM, Brooks S, Loewenstein J, Green S, Biesecker LG, Biesecker BB. Motivators for participation in a whole-genome sequencing study: implications for translational genomics research. Eur J Hum Genet 2011;19:1213-1217.

36. Facio $\mathrm{FM}$, Eidem $\mathrm{H}$, Fisher $\mathrm{T}$, et al. Intentions to receive individual results from whole-genome sequencing among participants in the ClinSeq study. Eur J Hum Genet 2013:21:261-265.

37. Patenaude AF, Dorval M, DiGianni LS, Schneider KA, Chittenden A, Garber JE. Sharing BRCA1/2 test results with first-degree relatives: factors predicting who women tell. J Clin Oncol 2006;24:700-706.

38. Bowen DJ, Bourcier E, Press N, Lewis FM, Burke W. Effects of individual and family functioning on interest in genetic testing. Community Genet 2004;7: 25-32. 